\title{
Anisotropic Plastic Behavior of Additively Manufactured PH1 Steel
}

Wenqi Liu, Zinan Li, Sven Bossuyt, Antti Forsström, Zaiqing Que, Roy Björkstrand, Mika Salmi, Jouni Partanen and Junhe Lian

Wenqi Liu. Advanced Manufacturing and Materials, Department of Mechanical Engineering, Aalto University, 02150 Espoo, Finland Zinan Li. Advanced Manufacturing and Materials, Department of Mechanical Engineering, Aalto University, 02150 Espoo, Finland Sven Bossuyt. Advanced Manufacturing and Materials, Department of Mechanical Engineering, Aalto University, 02150 Espoo, Finland

Antti Forsström. Advanced Manufacturing and Materials, Department of Mechanical Engineering, Aalto University, 02150 Espoo, Finland

Zaiqing Que. VTT Technical Research Centre of Finland Ltd, 02044 Espoo, Finland

Roy Björkstrand. Advanced Manufacturing and Materials, Department of Mechanical Engineering, Aalto University, 02150 Espoo, Finland

Mika Salmi. Advanced Manufacturing and Materials, Department of Mechanical Engineering, Aalto University, 02150 Espoo, Finland

Jouni Partanen. Advanced Manufacturing and Materials, Department of Mechanical Engineering, Aalto University, 02150 Espoo, Finland

Junhe Lian. Advanced Manufacturing and Materials, Department of Mechanical Engineering, Aalto University, 02150 Espoo, Finland

Impact and Crashworthiness Lab, Department of Mechanical Engineering, Massachusetts Institute of Technology, 77 Massachusetts Avenue, Cambridge,MA 02139-4307, USA

Corresponding author: Junhe Lian. E-mail address: junhe.lian@aalto.fi

Abstract. Metals made by additive manufacturing (AM) have intensely augmented over the past decade for customizing complex structured products in the aerospace industry, automotive, and biomedical engineering. However, for AM fabricated steels, the correlation between the microstructure and mechanical properties is yet a challenging task with limited reports. To realize optimization and material design during the AM process, it is imperative to understand the influence of the microstructural features on the mechanical properties of AM fabricated steels. In the present study, three material blocks with $120 \times 25 \times 15 \mathrm{~mm}^{3}$ dimensions are produced from PH1 steel powder using powder bed fusion (PBF) technology to investigate the anisotropic plastic deformation behavior arising from the manufacturing process. Despite being identical in geometrical shape, the manufactured blocks are designed distinguishingly with various coordinate transformations, i.e. alternating the orientation of the block in the building direction ( $\mathrm{z}$ ) and the substrate plate ( $\mathrm{x}, \mathrm{y})$. Uniaxial tensile tests are performed along the length direction of each specimen to characterize the anisotropic plastic deformation behavior. The distinctly anisotropic plasticity behavior in terms of strength and ductility are observed in the AM PH1 steel, which is explained by their varied microstructure affected by the thermal history of blocks. It could also be revealed that the thermal history in the AM blocks is influenced by the block geometry even though the same process parameters are employed.

Keywords. Additive Manufacturing, Martensitic Stainless Steel, Digital Image Correlation, Geometry Design, Plastic Anisotropy

\section{Introduction}

Additive manufacturing (AM) is one of the strongest driving forces to shorten the material/product development cycle in Industry 4.0, as it builds complex-shaped products by adding material layer-upon-layer from the raw materials, skipping the long production line in conventional manufacturing [1] and to finish the product directly on the market $[2,3]$. During fabricating customized products with advanced attributes by AM, rapid heating above the melting 
temperature, and solidification of the molten material occurs numerous times as the re-heating/re-cooling processes. Additively manufactured metal components are subjected to thermal treatment cycles [4]. Thus, the microstructure of an AM part can result in a large difference from the one by conventional manufacturing processes e.g. casting, or forging. The observed microstructural features including the porosity, grain size, grain shape, crystallographic texture, etc. affect material mechanical properties in return. Therefore, it is important to bridge the correlation between microstructure and mechanical properties, and understand the influence of the corresponding AM processing parameters, to realize process optimization and materials design.

Selective laser melting (SLM) is one representative of the powder bed fusion (PBF) technology. Studies have been performed previously in AM technique on many metals. Kok et al [6] carried out a critical review on experimentally investigated microstructure and mechanical properties and pointed out that the increased heterogeneity and defects in AM microstructure would bring profound anisotropy behavior, compared to their conventionally manufactured counterparts [7-9]. It was noted that the epitaxial columnar grain is a common microstructure morphology in AM metals, and the grain long axis is normally along the building direction (BD) [10], which causes the plastic anisotropy and even offers possible damage initiation site. For AM steels, the existing studies mainly focus on the austenite stainless steel, and the report on the AM martensite steel in terms of the anisotropic investigation is still blank in the literature, especially on the fine-scale in-depth investigations of the relationship between microstructural heterogeneities and their anisotropic mechanical properties.

This study focuses on the systemically characterization and analyses of the anisotropic plasticity behavior of a precipitation hardening stainless steel (PH1) by AM. The progress starts from geometry design and specimen manufacturing of the AM PH1 steel, followed by experiments on mechanical property characterization, mainly the anisotropic plastic responses. Section 2 gives an overview of AM PH1 steel production including specimen geometry design, material chemical composition, material production, and specimen manufacturing procedure. Section 3 introduces the lab-level mechanical testing techniques, i.e. the tensile testing coupling digital image correlation (DIC) method including the sample preparation, testing setup, and post-process for data analysis. Section 4 is the preliminary mechanical testing results and discussion.

\section{Material production}

\subsection{Material printing layout}

Analogously to traditional manufactured materials, e.g. the rolling direction-transverse direction-normal direction coordinate system of cold-rolled metals, the anisotropic responses of AM materials are investigated in terms of the different directions in the manufacturing space as well. For AM materials, the manufacturing space is formed by the building direction along the z-axis and the building plate ( $x-y$ plane), which is composed of the recoater direction (RD) along the $\mathrm{x}$-axis and transverse direction (TD) along the y-axis. Similar to rolling direction of rolled materials, the building direction is normally regarding as the focused direction of AM materials. There are two methods to manufacture the anisotropic specimens. The first one is similar to the traditional method by producing large material plates or sheets and then cutting targeted specimens along aimed investigation directions. The second method is directly producing blocks or specimens with closed dimensions for testing along varying investigation directions. In this study, the second method was chosen, and the anisotropic mechanical responses were characterized by the uniaxial tensile testing. The outline of the tensile specimen was $110 \mathrm{~mm} \times 20 \mathrm{~mm} \times 1.5 \mathrm{~mm}$. Considering 3-5 parallel mechanical tests for each loading condition and the allowance for the finish, the blocks with dimensions of $120 \mathrm{~mm}$ $\times 25 \mathrm{~mm} \times 15 \mathrm{~mm}$ were printed. The longest dimension is printed along the focused direction. The investigation directions are building direction, transverse direction, and recoater direction, marked with BD, TD, and RD respectively, as shown in Fig. 1. 


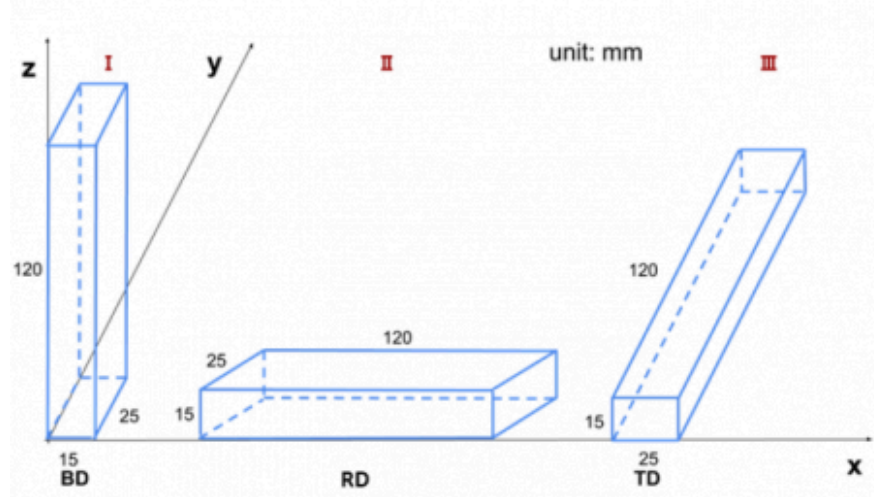

(a)

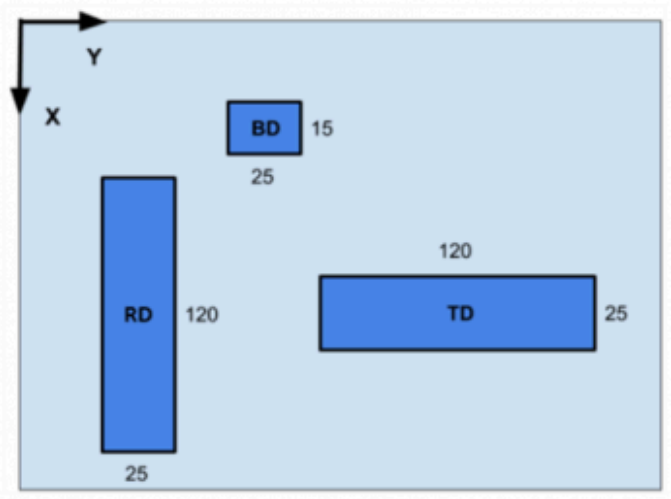

(b)

(a) 3D sketch of the printing plan, (b) 2D projection of the AM specimens on the building plate.

Fig. 1 Layout of AM PH1 specimens (unit: $\mathrm{mm}$ ).

\subsection{Material composition}

The samples were fabricated from the pre-alloyed stainless steel PH1 fine powder provided by Electro Optical System (EOS) GmbH, Germany. The nominal composition (in wt.\%) of the powder is shown in Table 1. The relative density of powder raw material is approximate $100 \%\left(7.8 \mathrm{~g} / \mathrm{cm}^{3}\right)$.

Table 1 Chemical composition of PH1 powder, wt.\%

\begin{tabular}{cccccccccc}
\hline Element & $\mathrm{Fe}$ & $\mathrm{C}$ & $\mathrm{Si}$ & $\mathrm{Mn}$ & $\mathrm{Cr}$ & $\mathrm{Ni}$ & $\mathrm{Cu}$ & $\mathrm{Mo}$ & $\mathrm{Nb}$ \\
\hline Min & Bal. & - & - & - & 14.0 & 3.5 & 2.5 & - & 0.15 \\
Max & Bal. & 0.07 & 1.00 & 1.00 & 15.5 & 5.5 & 4.5 & 0.5 & 0.45 \\
\hline
\end{tabular}

\subsection{Specimen manufacturing}

The material was printed by an EOS M290_400W machine. The rotating scanning strategy was employed with $67^{\circ}$ rotation in each layer. The base plate temperature was set around $100{ }^{\circ} \mathrm{C}$. A consistent production parameter set recommended by EOS was employed for all printed PH1 blocks. After printing, no additional heat treatment process was applied, therefore, the original printed microstructure is preserved. The blocks were wire-cut by electrical discharge machining (EDM) to obtain the final tensile specimen geometry according to the drawing in Fig. 2. The dogbone outline is used for the uniaxial tensile testing with a gauge length of $30 \mathrm{~mm}$, a width of $6 \mathrm{~mm}$, and a thickness of $1.5 \mathrm{~mm}$. 


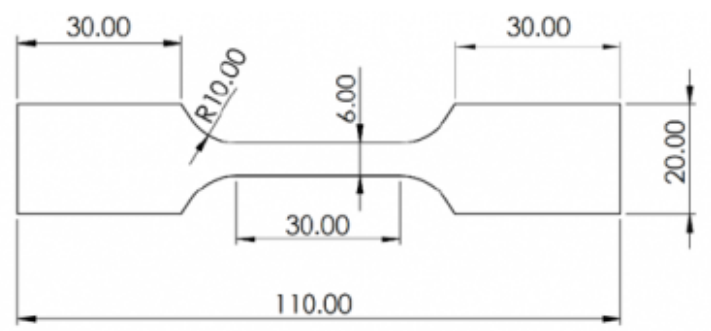

Fig. 2 Tensile test sample geometry. (unit: $\mathrm{mm}$ )

\section{Mechanical testing}

\subsection{Specimen preparation}

The digital image correlation (DIC) technique was used to measure the displacement during the tensile testing. The full-field displacement could be obtained by the optical DIC system. High surface quality is required for the DIC tensile specimens. After EDM, if the specimen surface flatness was not good enough, additional grinding and polishing preparation was conducted. The observed specimen surface is lacquered with an even coating layer of white paints as background to ensure the image quality. Besides, to improve the spatial resolution and displacement sensitivity of the DIC measurement, a pre-designed optimal feature pattern with 1800 dpi resolution [11] was printed on a tattoo paper. Then the pre-printed transparent tattoo paper further adhered to the specimen surface. The finally prepared specimen is shown in Fig. 3 (a). A detailed tattoo paper pattern can be seen in Fig. 3 (b) and with a zoom-in of the localized area in (c).

\subsection{Tensile testing setting up}

The uniaxial tensile testing was performed at room temperature with a Zwick/Roell Z020 screw-driven tensile testing machine, which has a maximum load capacity of $20 \mathrm{kN}$. To start the tensile testing, the specimen of AM PH1 was gripped at each end and stretched along its length direction at a constant crosshead velocity in the universal tensile test machine until it fractures. The applied load and full-field displacement at the uniform deformation zone were measured using a load cell and an optical DIC system, respectively. The chosen constant crosshead velocity was $0.9 \mathrm{~mm} / \mathrm{min}$, and for the specimen gauge length of $30 \mathrm{~mm}$, it corresponded to the quasi-static strain rate of $5 \times 10^{-4} \mathrm{~s}^{-1}$ before necking. 3 parallel tests were conducted for each loading condition. 


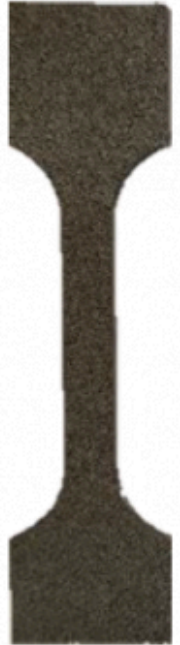

(a)

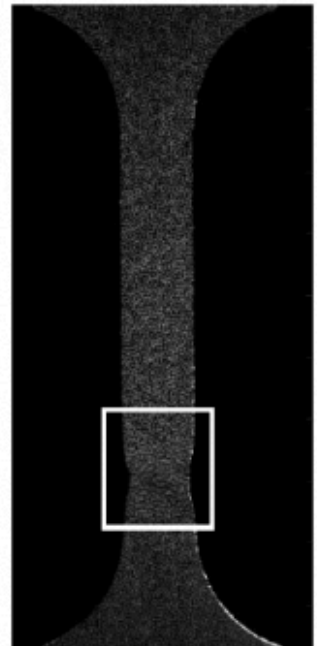

(b)

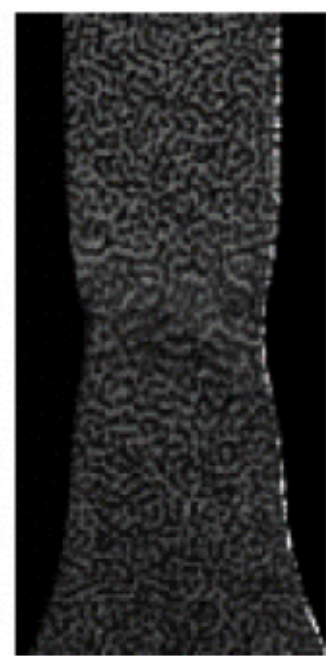

(c)

(a) Before the tensile testing, (b) Last deformation image before fracture, (c) the zoom-in of the highlighted area in (b) with a focus on the severe localization.

Fig. 3 Specimens with 1800 dpi DIC pattern.

The testing set-up is shown in Fig. 4. Lighting conditions were improved by utilizing indirect diffuse lighting, which minimized reflections from the material surface. A spotlight was placed behind the specimen and the light is reflected from two curved curtains made of projector screen canvas, located in front of the specimen. Observations by the DIC camera were done from an opening between the curtains. The DIC camera detector had a resolution of 20 pixels $/ \mathrm{mm}$. The device recorded pictures at a detection rate of $2 \mathrm{~Hz}$ during testing. Fig. 3 (b) shows an example of the DIC image of the last deformation frame before the final fracture. It can be seen that the pattern on the tattoo paper is still consistent even if there is strong localization. 


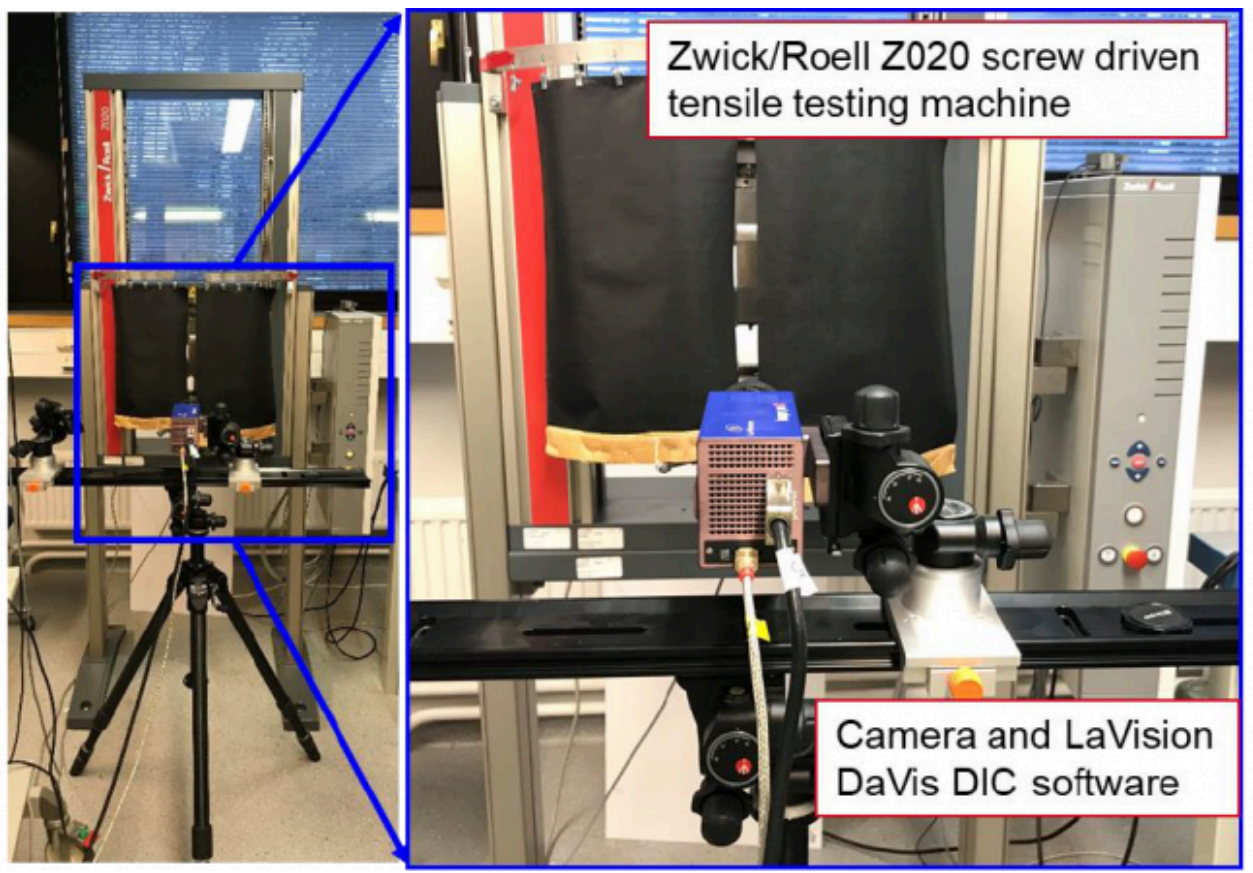

Fig. 4 Uniaxial tensile testing set-up with DIC system and indirect diffuse lighting.

\subsection{Post-process for data evaluation}

In terms of testing result evaluation procedure, from the measured force-displacement data, both the engineering stress-strain curves and the flow curves can be calculated, as well as the Lankford coefficients (r-values) evolution curves. With the measured force F by the load cell, displacement $\Delta l$ by DIC and initial specimen dimensions (length $l_{0}$ , width $w_{0}$, and thickness $t_{0}$ ), the engineering stress $\sigma_{\mathrm{E}}$, engineering strain $\varepsilon_{\mathrm{E}}$, true stress $\sigma_{\mathrm{T}}$, and true strain $\varepsilon \mathrm{T}$ are calculated as follows:

$$
\begin{gathered}
\sigma_{\mathrm{E}}=\frac{F}{w_{0} \cdot t_{0}} \\
\varepsilon_{\mathrm{E}}=\frac{\Delta l}{l_{0}} \\
\sigma_{\mathrm{T}}=\sigma_{\mathrm{E}}\left(1+\varepsilon_{\mathrm{E}}\right) \\
\varepsilon_{\mathrm{T}}=\ln \left(1+\varepsilon_{\mathrm{E}}\right)
\end{gathered}
$$

The true plastic strain along the elongation direction, i.e. length, is calculated according to equation (5) [12]:

$$
\varepsilon_{\mathrm{T}, \mathrm{p}, \mathrm{l}}=\ln \left[\frac{l_{0}+\Delta l}{l_{0}}-\frac{F}{S_{0} \cdot m_{E}}\right]
$$


along the transverse direction, i.e. width, is calculated according to equation (6) with the material Poisson's ratio $v$ :

$$
\varepsilon_{\mathrm{T}, \mathrm{p}, \mathrm{w}}=\ln \left[\frac{w_{0}-\Delta w_{0}+w_{0} \cdot v \cdot \frac{F}{S_{0} m_{\mathrm{E}}}}{w_{0}}\right]
$$

According to the constant volume rule during the plastic deformation, the true plastic strain along the third direction, i.e. thickness, is calculated according to equation (7):

$$
\varepsilon_{\mathrm{T}, \mathrm{p}, \mathrm{t}}=-\left(\varepsilon_{\mathrm{T}, \mathrm{p}, \mathrm{l}}+\varepsilon_{\mathrm{T}, \mathrm{p}, \mathrm{w}}\right)
$$

The Lankford coefficient, i.e. r-value, is defined as the ratio of the true plastic strain of width and thickness directions:

$$
r=\varepsilon_{\mathrm{T}, \mathrm{p}, \mathrm{w}} / \varepsilon_{\mathrm{T}, \mathrm{p}, \mathrm{t}}
$$

The results are shown in Section 4.

\section{Results and discussion}

The anisotropic behavior of AM PH1 steel is characterized by the engineering stress-strain, the true stress-strain, and the r-values, as shown in Fig. 5 and Fig. 6. The parallel tests along each direction show good repeatability on the results. The AM PH1 steel has a yield strength (YS) close to 900 MPa and the ultimate tensile strength (UTS) higher than 1000 MPa along all loading directions. Meanwhile, a large deviation in uniform and fracture elongation can be observed when the loading direction varies. The fracture elongation can reach around 10\% along RD/TD but only around 7\% along BD. The r-value of AM PH1 steel is always smaller than 1 and increases with strain evolution, which means, the plastic deformation along the specimen thickness directions is always larger than it along the width directions, no matter which manufacturing direction of the specimen is.

Distinct anisotropic response of the AM PH1 steel can be observed from these results. Generally, the RD and TD results are similar or almost overlapping with each other, especially for the stress-strain responses. This is caused by the rotating scanning strategy. During the material production, the laser scanning direction (SD) is not always along the $\mathrm{RD}$, instead, it rotates $67^{\circ}$ between each layer to avoid spattering and improve the quality of printed specimens. Therefore, at the macroscopic level, with the specimen thickness much larger than the scanning layer thickness, the defined RD and TD shall be identical. More generally, the macroscopic mechanical property along any direction on the RD-TD plane shall be consistent, which is reflected by the stress-strain curves and the r-values of the materials tested along RD and TD.

Meanwhile, the curves along BD is far away from the curves of RD and TD, especially in terms of the elongation or strain behavior. The similar yield strength values along different loading directions can be observed. However, the UTS along BD is more than $100 \mathrm{MPa}$ higher than that along $\mathrm{RD} / \mathrm{TD}$, which is also related to the anisotropic strain hardening behavior $[13,14]$. The BD specimens have the shortened uniform and fracture elongation compared to the 
$\mathrm{RD} / \mathrm{TD}$ specimens, and lower strength. The evolution of the anisotropic behavior is also shown for both stresses and r-values, as presented in Fig. 6. For the true stress, the difference between BD and RD/TD is reduced. A hypothesis is proposed to explain the anisotropic behavior of the investigated AM PH1 steel. The reason for the different macroscopic mechanical properties shall be related to the microstructure information, which is strongly affected by the production process. Since there is no post AM heat treatment was performed in this study, the microstructure of specimens is directly resulted from the additive manufacturing process. As aforementioned, a consistent production parameter set was employed for all printed PH1 blocks in this study. However, due to the different block geometries, i.e. dimensions of the printed blocks (Fig.1), the thermal history within these blocks might differ from each other. For instance, as the BD block has a relatively smaller cross-section (on RD-TD plane, $15 \mathrm{~mm} \times 25 \mathrm{~mm}$ ), a shorter laser scanning path is expected on each layer. The material will go through more frequent repeats or even continuously heating. The continuously thermal effect time is increased, which might result in the continuously abnormal grain growth along the BD. The big and heterogeneous grain size will lead to reduced ductility. While for the RD and TD specimens, the cross-section on the RD-TD plane is much larger $(120 \mathrm{~mm} \times 25 \mathrm{~mm})$. Therefore, the thermal effect along the BD might be also reduced, which might lead to the smaller grain size in these specimens and the grain size distribution shall also be more homogeneously compared to the BD block. The smaller and more homogenous grain size distribution shall take responsibility for the higher strength and distinctly increased uniform and fracture elongation of the RD/ TD specimens. In addition, the different thermal field and history would also bring different grain shape and crystal orientation distribution. For example, the continuously abnormal grain growth along the BD might bring an extremely small grain shape aspect ratio (length ratio between the shortest axis and the longest axis) and strong texture in the BD specimen. Due to the technique's limitation, thermal history cannot be recorded during production. The microstructure analysis is planned to verify the proposed hypotheses. The electron backscatter diffraction (EBSD) measurement will be carried out to analyze the grain size, shape, and orientation distributions, which shall be helpful to reveal the reason for the macroscopic anisotropic properties of AM PH1.

Furthermore, more fine-scale in-depth investigations of the relationship between microstructural heterogeneities and their anisotropic mechanical properties shall be carried out by the numerical simulation approach. An artificial 3D representative volume element (RVE) coupling crystal plasticity (CP) method [15] can be employed for bridging the microstructure and anisotropic behavior. The CP parameters can be calibrated based on the reference material microstructure and flow curve along BD. The numerical approach including models and parameters can be validated by tensile response along other loading directions. With the calibrated CP model and parameters, a series of RVEs with variable microstructural features can be generated as a virtual laboratory to reveal the anisotropic behavior in terms of AM microstructure. Moreover, post AM heat treatment can be conducted to adjust the final microstructure. Therefore, a future study could be expected on the influence of heat treatment on plastic anisotropy.

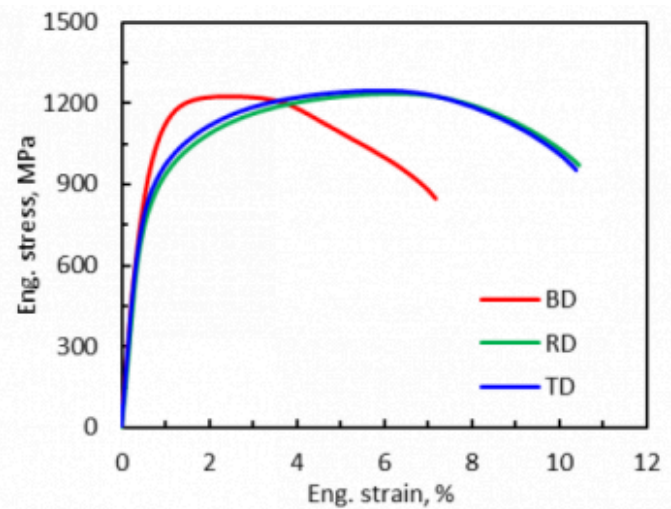

(a)

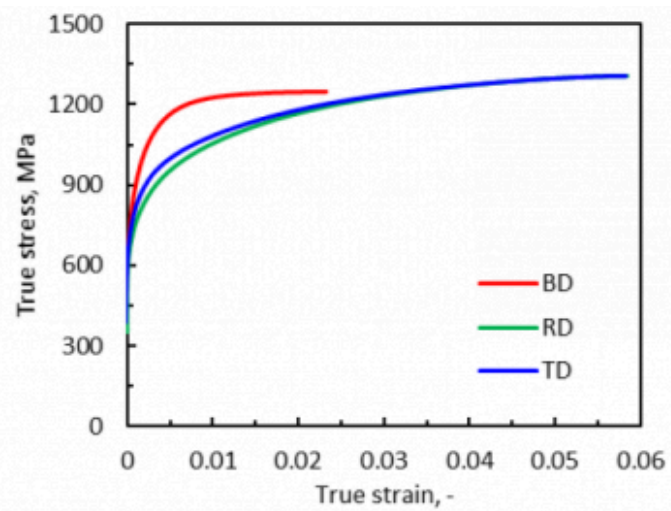

(b) 
Fig. 5 Stress-strain responses of AM PH1 steel along BD, RD, and TD uniaxial loading.

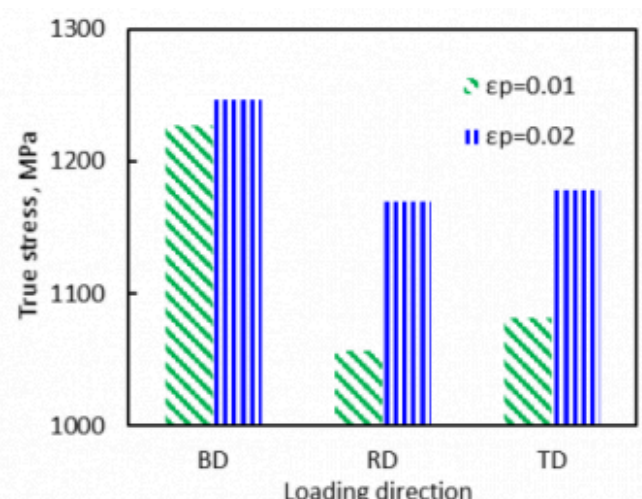

(a)

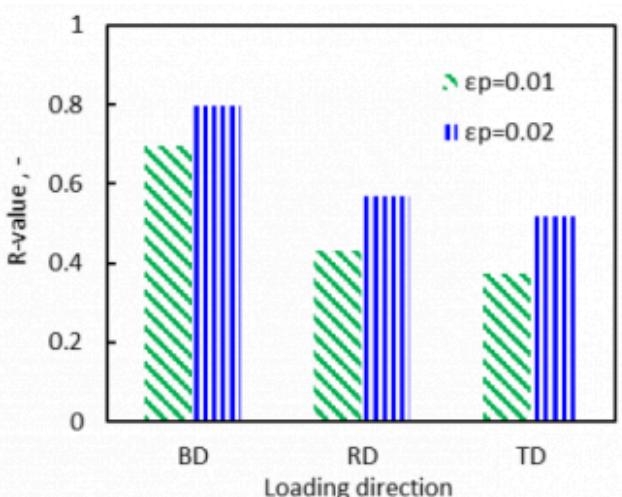

(b)

(a) True stress, (b) R-value.

Fig. 6 Anisotropic responses of AM PH1 steel along BD, RD, and TD uniaxial loading at the true plastic strain of 0.01 and 0.02 .

\section{Conclusions}

In this study, the anisotropic behavior of additive manufacturing PH1 stainless steel was investigated. Specimens were produced and elongated along the building direction, recoater direction, and transverse direction to study the plastic anisotropy behavior of the materials. With the same processing parameters, similar tensile properties are found for the samples tested along recoater and transverse directions; however, very distinct behavior is discovered for the test along the building directions. A hypothesis is proposed to explain the macroscopic anisotropic behavior, that the printing geometry also influences the grain size, shape, and texture of the AM materials. Therefore, a detailed microstructure analysis process is designed as an on-going study. Furthermore, the numerical laboratory shall be built up to obtain the quantitative relation between the AM microstructure and mechanical properties. The post AM heat treatment effect on final microstructure and anisotropic behavior can also be considered in the future study.

\section{Acknowledgments}

The authors gratefully acknowledge Mr Janne Peuraniemi and Mr Kim Widell from the Advanced Manufacturing and Materials group of the Department of Mechanical Engineering at Aalto University for their support on material manufacturing and mechanical testing.

\section{Bibliography}

[1] Sossou G, Demoly F., Montavon G., Gomes S. An additive manufacturing oriented design approach to mechanical assemblies. Journal of Computational Design and Engineering, 2018;5(1):3-18.

[2] Calle MAG, Salmi M, Mazzariol LM, Kujala P. Miniature reproduction of raking tests on marine structure: Similarity technique and experiment. Engineering Structures, 2020;212:110527.

[3] Calle MAG, Salmi M, Mazzariol LM, Alves M, Kujala P. Additive manufacturing of miniature marine structures for crashworthiness verification: Scaling technique and experimental tests. Marine Structures, 2020;72:102764. 
[4] Beese AM. Microstructure and mechanical properties of am builds. Thermo-Mechanical Modeling of Additive Manufacturing, 2018:81-92.

[5] Lewandowski JJ, Seifi M. Metal additive manufacturing: A review of mechanical properties. Annual Review of Materials Research, 2016;46(1):151-186.

[6] Kok Y, Tan XP, Wang P, Nai MLS, Loh NH, Liu E, Tor SB. Anisotropy and heterogeneity of microstructure and mechanical properties in metal additive manufacturing: A critical review. Materials \& Design, 2018;139:565-586.

[7] Motaman SAmir H, Roters F, Haase C. Anisotropic polycrystal plasticity due to microstructural heterogeneity: A multi-scale experimental and numerical study on additively manufactured metallic materials. Acta Materialia, $2020 ; 185: 340-369$.

[8] Wang YM, Voisin T, McKeown JT, Ye J, Calta NP, Li Z, Zeng Z, Zhang Y, Chen W, Roehling TT, Ott RT, Santala MK, Depond Philip J, Matthews MJ, Hamza AV, Zhu T. Additively manufactured hierarchical stainless steels with high strength and ductility. Nature Materials, 2018;17(1):63-71.

[9] Song B, Zhao X, Li S, Han C, Wei Q, Wen S, Liu J, Shi Y. Differences in microstructure and properties between selective laser melting and traditional manufacturing for fabrication of metal parts: A review. Frontiers of Mechanical Engineering, 2015;10(2):111-125.

[10] Takaichi A, Suyalatu, Nakamoto T, Joko N, Nomura N, Tsutsumi Y, Migita S, Doi H, Kurosu S, Chiba A, Wakabayashi $\mathrm{N}$, Igarashi Y, Hanawa T. Microstructures and mechanical properties of co-29cr-6mo alloy fabricated by selective laser melting process for dental applications. Journal of the Mechanical Behavior of Biomedical Materials, 2013;21:67-76.

[11] Bossuyt S. Optimized patterns for digital image correlation. Proceedings of the 2012 Annual Conference on Experimental and Applied Mechanics, Volume 3: Imaging Methods for Novel Materials and Challenging Applications, 2013.

[12] An YG, Vegter H, Melzer S, Romano Triguero P. Evolution of the plastic anisotropy with straining and its implication on formability for sheet metals. Journal of Materials Processing Technology, 2013;213(8):1419-1425.

[13] Lian J, Shen F, Jia X, Ahn D-C, Chae D-C, Münstermann S, Bleck W. An evolving non-associated hill48 plasticity model accounting for anisotropic hardening and $r$-value evolution and its application to forming limit prediction. International Journal of Solids and Structures, 2018;151:20-44.

[14] Xie Q, Van Bael A, An YG, Lian J, Sidor JJ. Effects of the isotropic and anisotropic hardening within each grain on the evolution of the flow stress, the r-value and the deformation texture of tensile tests for aa6016 sheets. Materials Science and Engineering: A, 2018;721:154-164.

[15] Liu W, Lian J, Aravas N, Münstermann S. A strategy for synthetic microstructure generation and crystal plasticity parameter calibration of fine-grain-structured dual-phase steel. International Journal of Plasticity, 2020;126:102614.

PDF automatically generated on 2021-05-20 06:22:29

Article url: https://popups.uliege.be/esaform21/index.php?id=4236

published by ULiège Library in Open Access under the terms and conditions of the CC-BY License (https://creativecommons.org/licenses/by/4.0) 